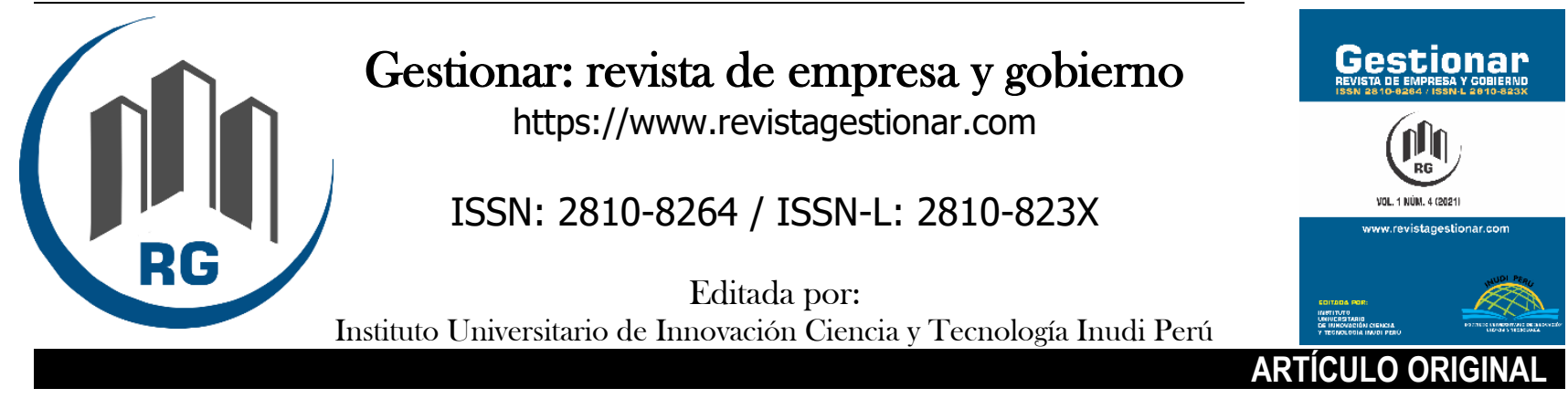

\title{
El método cualitativo y su aporte a la investigación en las ciencias sociales
}

\author{
The qualitative method and its contribution to research in the social sciences \\ 0 método qualitativo e sua contribuiç̧ão para a pesquisa em ciências sociais \\ Víctor Guzmán ${ }^{1}$ \\ Corporación Universitaria Iberoamericana, Bogotá - Bogotá, Colombia \\ (iD https://orcid.org/0000-0002-6051-3153 \\ vaguzmanbrand@gmail.com
}

DOI: https://doi.org/10.35622/j.rg.2021.04.002

Recibido: 14/09/2021 Aceptado: 31/12/2021 Publicado: 31/12/2021
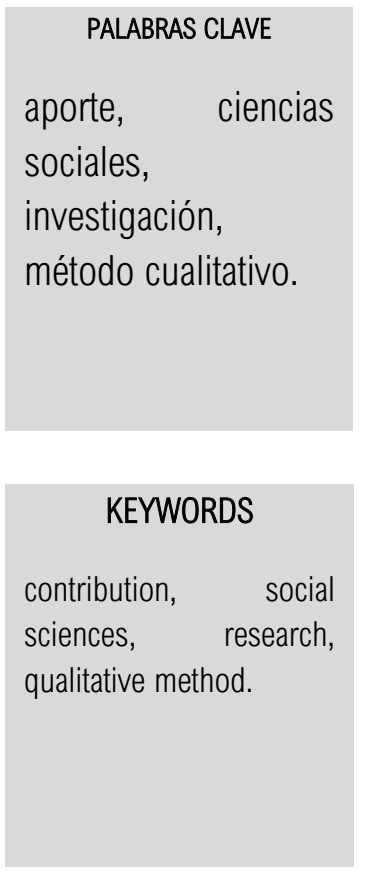

PALAVRAS-CHAVE
RESUMEN. El presente artículo de reflexión tiene como finalidad dar a conocer las características y bondades que tiene la investigación de corte cualitativo como herramienta clave para la implementación de investigaciones en el campo disciplinar de las ciencias sociales. Para lo cual se utiliza la metodología de enfoque documental, mediante la exploración de fuentes primarias y secundarias de información que permitan ahondar en la teoría y postulados claves, pretendiendo llegar al punto de tomar la argumentación de los autores más reconocidos en los parámetros de la metodología de la investigación. Como resultado podemos encontrar que la investigación cualitativa es un proceso fundamental con que cuenta el investigador para comprender la realidad presente en el contexto, por lo tanto, este paradigma extrae la esencia de las experiencias de las personas y expone los aspectos más arraigados.

ABSTRACT. The purpose of this article for reflection is to present the characteristics and benefits of qualitative research as a vital tool for implementing research in the disciplinary field of the social sciences. For which the documentary approach methodology is used, by exploring primary and secondary sources of information that allow delving into the theory and key postulates, trying to reach the point of taking the argumentation of the most recognized authors in the parameters of the methodology of the investigation. As a result, we can find that qualitative research is a fundamental process that the researcher has to understand the reality present in the context. Therefore, this paradigm extracts the essence of people's experiences and exposes the most deeply rooted aspects.

RESUM0. 0 objetivo deste artigo de reflexão é apresentar as características e benefícios da pesquisa qualitativa como ferramenta fundamental para a implementação da pesquisa no campo disciplinar das ciências sociais. Para o qual se utiliza a metodologia de abordagem documental, através da exploração de fontes primárias e secundárias de informação que permitam aprofundar a teoria e postulados-chave, tentando chegar ao ponto de levar a argumentação dos autores mais

1Psicólogo Especialista en Desarrollo Integral de la Infancia y Adolescencia. Correspondencia: vaguzmanbrand@gmail.com 
contribuição, ciências sociais, pesquisa, método qualitativo. reconhecidos nos parâmetros da metodologia de a investigação. Como resultado, podemos constatar que a pesquisa qualitativa é um processo fundamental que o pesquisador possui para compreender a realidade presente no contexto, portanto, este paradigma extrai a essência das experiências das pessoas e expõe os aspectos mais profundamente enraizados.

\section{INTRODUCCIÓN}

El proceso de investigación presenta diferentes métodos y diseños que sirven de guía al investigador para lograr desentramar la situación problema que ha descrito en su proyecto, por lo tanto, es indispensable que conozca las estructuras que le ayudaran de soporte. Es así como cada modelo persigue fines diferentes y por esta razón debe tenerse claro los objetivos y el fin de cada página en la investigación, entonces, la importancia de escoger uno o varios de estos esquemas está orientada a la relevancia del problema en investigación, así que su relevancia no está supeditada al modelo, puesto que este se establece en relación con las especificaciones del problema analizado y el tipo de resultado que se quiere alcanzar (Pimienta et al., 2012).

Por otro lado, cualquier metodología que se escoja como pilar del proceso investigativo está acompañada de un paradigma el cual "es un conjunto de concepciones y premisas acerca del mundo y los métodos y técnicas que se consideran apropiadas para conocerlo e investigarlo"(Hernández \& Mendoza, 2018, p. 5). Es así que radica su importancia en la forma de observar el objeto de estudio y su perspectiva metodológica, además esta teoría tiene como objetivo disponer la relación en las que circunstancias se manifiestan sobre las reconocidas premisas, por lo cual de adopta el entorno como fórmula para describir la vía a la hora de explicar cada una de las predicciones, por esta razón se llega a reflexionar, inferir y predecir por medio del proceso otros eventuales sucesos (Bautista, 2013).

Una de las grandes dificultades del investigador es tener claridad y profundizar sobre la ruta metodología que utilizara en su proceso investigativo, en cuanto a que la literatura existente es muy extensa y genera muchos interrogantes, además al disponer con un gran número de paradigmas, métodos y estrategias para realizar su proyecto este se limita hacia aspectos generales, por lo tanto "es una falta ética enfrentar una investigación sin el debido conocimiento metodológico y estratégico acorde tanto a la problemática como a las particularidades del grupo social abordado" (Bautista, 2013, p. 24). Por esta razón, es indispensable que cuando se seleccione cualquier metodología esta debe orientarnos a conocer la realidad de forma asertiva. Es así como es recomendado que el método escogido tenga un estudio previo sobre los fines investigativos "puesto que el valor se encuentra en saber acudir a ellos en razón de las metas perseguidas” (Bautista, 2013, p. 15).

Ahora bien, la investigación cualitativa es una visión del mundo que nos rodea que ha tenido a lo largo de la historia muchos opositores, pero cada vez en su recorrido acumula más seguidores y defensores de sus procesos metodológicos, en esta se plasma una intensión para salirse de la rigidez positivista en relación con diversos problemas sociales, donde emplea un proceso interpretativo de forma personal con el propósito de comprender la realidad. Entonces la metodología cualitativa como lo manifiesta tiene la misma validez que la metodología cuantitativa y su divergencia radica en su objetivo, capacidad de descubrimiento y contenido de la estrategia de cada una, por lo cual se aplican en contextos y circunstancias diferentes, en atención a lo cual "el acierto del investigador depende no de la metodología que utiliza sino del acierto en aplicarla en aquellos casos específicos para los que está más adaptada" (Ruiz, 2012, p. 17). 
Con relación a lo anterior el significado de la palabra metodología nos hace referencia a el como hacer, es así como este marco es una agrupación de ideas que construyen una estrategia y producen un proceso que sirve de vehículo para el camino en la investigación. Entonces, en la planificación de la metodología, "un investigador asocia estos puntos de vista y estas perspectivas con las técnicas de trabajo empleadas. Este conjunto debe formar un todo coherente y ordenado, donde las decisiones metodológicas derivan de las posturas epistemológicas" (Gómez et al., 2010, p. 95).

\section{MÉTODO}

Para el presente artículo de reflexión se implementó la búsqueda y rastreo bibliográfico en relación con el método documental, el cual parte de la búsqueda bibliográfica, caracterizándose por la exploración en fuentes primarias o de primera mano y secundarias o de segunda mano de información utilizadas en el ámbito académico siendo las más conocidas los libros y artículos en revistas científicas entre otras. En relación con la idea anterior al pretender extraer la información necesaria en las fuentes para el plan de trabajo, debe estar claro estos conceptos, por lo tanto, "las fuentes primarias son las evidencias originales, las fuentes secundarias son las informaciones proporcionadas por otros investigadores" (González, 2015, p. 3). Luego se debe consultar, organizar y especificar la información relevante y concreta consecuente a la temática en estudio.

Esta gran fuente que reviste de riqueza es entendida como un gran mundo de conocimiento y acumulan información veraz y confiable sobre la temática que exponen, siendo enormemente especializadas, adicionalmente estas fuentes están a la mano del investigador al poder ser consultadas en bibliotecas digitales y en páginas en internet. Por consiguientes la investigación documental conduce al investigador a plantear una primera búsqueda y recopilación de libros, documentos, artículos y expedientes que han sido publicados sobre la temática en estudio siempre teniendo en cuenta que esta exploración se debe hacer de lo general hacia lo particular lo cual nos conduce a proponer que las primeras actividades de exploración se produzcan de forma general en dirección al tema.

El análisis documental se efectúa de acuerdo a cinco etapas, en primer lugar, se realiza una búsqueda y organización de los documentos disponibles para esto se utiliza las bibliotecas virtuales, al igual de las bases de datos dispuestas en la internet, a su vez se dispone de libros impresos sobre la investigación cualitativa. En segundo lugar, se debe aplicar una clasificación de la información distinguida y concreta, en tercer lugar, aparece la selección y priorizando los documentos relevantes para el propósito de la investigación, en cuarto lugar, esta una lectura profunda sobre el contenido del material escogido de este se toma los elementos de análisis y se registran en los memos o notas marginales recogiendo los patrones, ideas, convergencias y toda aquella información que surge, en el quinto y último paso se efectúa una lectura cruzada y se comparan los documentos recolectados, no del contenido total de cada uno sino de la información descubierta al punto que se puede efectuar una síntesis comprensiva general (Sandoval, 2002).

\section{RESULTADOS Y DISCUSIONES}

Es claro que la investigación cualitativa es una mirada enfocada al análisis de los fenómenos, examinando a partir de la posición de los elementos presentes en el ambiente y vinculados a su contexto. Por este motivo esta 
metodología debe ser elegida cuando se pretenda conocer como los individuos presencian los sucesos y situaciones que están presenten a su alrededor, de igual forma se pretende profundizar en sus observaciones, interpretaciones y significados. En relación con esto la metodología de corte cualitativo para Baena (2017) parte de la idea "que el mundo social está constituido de significados y símbolos compartidos de manera intersubjetiva, razón por la cual su objetivo es la comprensión de esos significados y símbolos intersubjetivos tal como son expresados por las personas" (p.73). Por lo tanto, se trata de entender la ocurrencia de las realidades sociales, además de su estructura como origen de su comportamiento.

Es así como el enfoque es recomendable en el momento que el investigador al realizar el análisis bibliográfico observe que tiene poco estudio o cuando el hecho a investigar tenga gran trascendencia en la población, para poder encontrar sus detonantes y si es el caso proponer una solución. Dado esto la investigación cualitativa en relación con las ciencias sociales esta cimentada bajo las bases de comprender las acciones que producen los actos humanos y las razones por las cuales se realizan. Por lo cual según Cook y Reichardt (1995, como se citó en Hurtado, 2000) postulan que este método tiene "una concepción global fenomenológica, inductiva, estructuralista, subjetiva, orientada al proceso y propia de la antropología social"(p.5). Por consiguiente, la metodología es preferiblemente enfocado al estudio de las personas y como se desarrolla su comportamiento en el ambiente social, pero esta situación es variable y definitivamente inestable en cuando a que una opinión sobre un asunto puede ser transformada con el tiempo. Algunas características relevantes del método cualitativo son las siguientes:

1.El investigador 0 investigadora plantea un problema, pero no sigue un proceso definido claramente. Sus planteamientos iniciales no son tan específicos como en el enfoque cuantitativo y las preguntas de investigación no siempre se han conceptualizado ni definido por completo.

2. En la búsqueda cualitativa, en lugar de iniciar con una teoría y luego "voltear" al mundo empírico para confirmar si ésta es apoyada por los datos y resultados, el investigador comienza examinando los hechos en sí y en el proceso desarrolla una teoría coherente para representar lo que observa (Esterberg, 2002). Dicho de otra forma, las investigaciones cualitativas se basan más en una lógica y proceso inductivo (explorar y describir, y luego generar perspectivas teóricas). Van de lo particular a lo general. Por ejemplo, en un estudio cualitativo típico, el investigador entrevista a una persona, analiza los datos que obtuvo y saca conclusiones; posteriormente, entrevista a otra persona, analiza esta nueva información y revisa sus resultados y conclusiones; del mismo modo, efectúa y analiza más entrevistas para comprender el fenómeno que estudia. Es decir, procede caso por caso, dato por dato, hasta llegar a una perspectiva más general.

3. En la mayoría de los estudios cualitativos no se prueban hipótesis, sino que se generan durante el proceso y se perfeccionan conforme se recaban más datos. Son un resultado del estudio.

4. El enfoque se basa en métodos de recolección de datos no estandarizados ni predeterminados completamente. Tal recolección consiste en obtener las perspectivas y puntos de vista de los participantes (sus emociones, prioridades, experiencias, significados y otros aspectos más bien subjetivos). También resultan de interés las interacciones entre individuos, grupos y colectividades. El investigador hace preguntas más abiertas, recaba datos expresados a través del lenguaje escrito, verbal y no verbal, así como visual, los cuales describe, analiza y convierte en temas que vincula, y reconoce sus tendencias personales. Debido a ello, la preocupación directa del investigador se concentra en las vivencias de los participantes tal como fueron (o son) sentidas y experimentadas (Sherman \& Webb, 
2005). Patton (2011) define los datos cualitativos como descripciones detalladas de situaciones, eventos, personas, interacciones, conductas observadas y sus manifestaciones.

5. Así, el investigador cualitativo utiliza técnicas para recolectar datos, como la observación no estructurada, entrevistas abiertas, revisión de documentos, discusión en grupo, evaluación de experiencias personales, registro de historias de vida, e interacción e introspección con grupos 0 comunidades.

6. El proceso de indagación es más flexible y se mueve entre las respuestas y el desarrollo de la teoría. Su propósito consiste en "reconstruir" la realidad, tal como la observan los actores de un sistema social definido previamente. Es holístico, porque se precia de considerar el "todo" sin reducirlo al estudio de sus partes.

7. La aproximación cualitativa evalúa el desarrollo natural de los sucesos, es decir, no hay manipulación ni estimulación de la realidad (Corbetta, 2007).

8. La investigación cualitativa se fundamenta en una perspectiva interpretativa centrada en el entendimiento del significado de las acciones de seres vivos, sobre todo de los humanos y sus instituciones (busca interpretar lo que va captando activamente).

9. Postula que la "realidad" se define a través de las interpretaciones de los participantes en la investigación respecto de sus propias realidades. De este modo, convergen varias "realidades", por lo menos la de los participantes, la del investigador y la que se produce en la interacción de todos los actores. Además, son realidades que van modificándose conforme transcurre el estudio y son las fuentes de datos.

10. Por lo anterior, el investigador se introduce en las experiencias de los participantes y construye el conocimiento, siempre consciente de que es parte del fenómeno estudiado. Así, en el centro de la investigación está situada la diversidad de ideologías y cualidades únicas de los individuos.

11. Las indagaciones cualitativas no pretenden generalizar de manera probabilística los resultados a poblaciones más amplias ni obtener necesariamente muestras representativas; incluso, regularmente no pretenden que sus estudios lleguen a repetirse.

12. El enfoque cualitativo puede concebirse como un conjunto de prácticas interpretativas que hacen al mundo "visible", lo transforman y convierten en una serie de representaciones en forma de observaciones, anotaciones, grabaciones y documentos. Es naturalista (porque estudia los fenómenos y seres vivos en sus contextos o ambientes naturales y en su cotidianidad) e interpretativo, pues intenta encontrar sentido a los fenómenos en función de los significados que las personas les otorguen (Hernández et al., 2014, p. 9).

Profundizando sobre el concepto encontramos que Taylor y Bogdan (1984) propone que este modelo tiene unas características y está a su vez tiene unos atributos propios para estudiar y entender la realidad social basado en sus rasgos así:

1.Abordar la realidad de forma inductiva para comprenderla y explicarla, y no para construir o evaluar o contrastar modelos, hipótesis o teorías preconcebidos.

2. Estudiar a los sujetos y su realidad de forma holística y contextualizada en su momento y circunstancias. Las personas, los escenarios o los fenómenos no son reducidos a variables, sino dinámicos y con enfoque sistémico y holístico, es decir, como un todo. 
3. Exigir sensibilidad y conciencia por parte de los investigadores de los efectos que ellos mismos causan en las personas sujeto de estudio. Por ello, implica una interacción de un modo natural y no intrusivo con las personas y su realidad. En la recopilación de la información se sigue el modelo de una conversación normal, y no el de los cuestionarios o guías de entrevista de preguntas y respuestas. Así los investigadores cualitativos saben que, aunque ellos no pueden eliminar sus efectos sobre las personas y la realidad que estudian buscan reducir esa influencia al mínimo; especialmente, cuando interpretan la información recopilada referente al tema definido para el estudio.

4. Exigir a los investigadores comprender a las personas y su realidad dentro del marco de referencia de ellas mismas. Para ello, es esencial que el investigador experimente la realidad tal como otros la experimentan.

5. Exigir a los investigadores tener la capacidad de apartarse de sus propias creencias, perspectivas y predisposiciones; es decir, no dar nada por sobrentendido acerca de la realidad en estudio; asumir las diferentes perspectivas del sujeto de estudio como valiosas; no buscar la verdad o la moralidad en sentido clásico, sino una comprensión detallada de las perspectivas de las personas.

6. Ser conscientes de que los métodos mediante los cuales se estudia a las personas necesariamente influyen sobre el modo en que estas son entendidas. Hay que considerar que al reducir las palabras y sus actos a ecuaciones estadísticas se pierde de vista el aspecto humano de la vida social. En cambio, considera que estudiar a las personas mediante la interpretación de sus actos permite conocerlas en lo personal y experimentar lo que ellas sienten en sus luchas cotidianas en la sociedad.

7. Tener en cuenta que las personas y los escenarios son a su vez similares y únicos. Similares en el sentido de que toda persona y en cualquier escenario se hallan rasgos y procesos sociales de tipo general. Es decir, similares para las personas entre sí y los escenarios entre sí. Únicos porque cada persona y cada escenario tienen rasgos propios.

8. Reconocer que en la actualidad los métodos cualitativos requieren ser más rigurosos y estandarizados como los son otros enfoques investigativos. A este respecto, los investigadores requieren ser flexibles en cuanto al modo en que deben conducir sus estudios y seguir lineamientos orientadores y no reglas (citado en Bernal, 2010, pp. 73-74).

Ahora bien, según lo planteado por Hernández y Mendoza (2018) a la hora de construir el marco metodológico en primer lugar estaría el paradigma; luego, el enfoque, por ende, si este fuere fenomenológico o constructivista el método es cualitativo. Por último, se formula el problema de investigación en relación con el enfoque elegido. Por otro lado, el paradigma que acompaña al enfoque sea cualitativo o cuantitativo que elegimos para ser aplicado a la situación. Es descrito por Kuhn (1962, citado por Pimienta et al., 2012, p. 59) como "cada uno de estos modelos en que basa en un paradigma, es decir, en el conjunto de supuestos, postulados, concepciones de la realidad y juicios de valor que sirven de referencia a la investigación" lo cual nos puntualiza que investigar, los datos a recolectar de qué forma recolectarlos, analizarlos e interpretarlos. Por eso, en relación con el enfoque epistemológico que se acoja es fundamental tener la noción del paradigma científico que conteste de manera adecuada al método a seguir, porque cada uno persigue un modelo diferente. Según el diseño de investigación y las técnicas de recolección de datos, también la forma de examinar los datos y ser vinculados con la teoría (Bautista, 2013). De la misma forma Kuhn (1962) "sostenía que las disciplinas científicas maduras se apoyan en un paradigma que define lo que se estudia (relevancia de los fenómenos sociales), por qué se estudia (formulación de hipótesis explicativas) y cómo se estudia (con qué métodos)" (Della \& Keating, 2013, p. 15). 
Al entrar en juego las perspectivas teóricas como parte del proceso investigativo las cuales mantiene el objetivo el postular las condiciones en las cuales se debe desarrollar los supuestos, "tomando como contexto una explicación del medio idóneo para que se desarrollen las predicciones. A raíz de ésta, se pueden especular, deducir y postular mediante ciertas reglas o razonamientos, otros posibles hechos" (Bautista, 2013, p. 44). Para llegar al conocimiento existen diferentes rutas teóricas que nos permiten dilucidar sobre la realidad social, entre las que tenemos: el Interpretativismo, la Hermenéutica, la Fenomenología, el Interaccionismo Simbólico y la Teoría Crítica, donde éstas son el entramado que cubre el fruto de la investigación las cuales se explicar de forma clara así:

El Interpretativismo: Esta corriente teórica es usada principalmente en la indagación de los hechos sociales, esta se "basa en el ejercicio de la interpretación que es el acto por el que otorgamos determinado sentido a cierta realidad en la que se muestra alguna intencionalidad. Las realidades que pueden ser objeto de interpretación son aquellas que se estiman involucradas con la acción de algún sujeto" (Bautista, 2013, p. 46). Por lo tanto, su punto de acción en el actor social y busca entender su visión, centrando a la persona en su estudio, por consiguiente, es desde el con quien construye, interpreta y modifica la realidad.

La Hermenéutica: Este paradigma es un modo de compresión critica que consiste en analizar las relaciones humanas en el espacio de la razón de la vida humana, es así como trata de interpretar y resolver los motivos de los actos de las personas, donde su origen emana del estudio de textos. Entonces, lo que busca la hermenéutica es "interpretar los sentidos culturales que los investigadores construyen a partir de lo expresado por los informantes sobre un asunto determinado" (Bautista, 2013, p. 56).

La Fenomenología: Trata de representar la configuración de la experiencia como está concebida en la conciencia, dejando a un lado la teoría, deducción o suposiciones de otras disciplinas. Es según su fundador Edmund Husserl "es una permanente actividad de búsqueda y de mediación sobre lo considerado evidente, un puro dudar frente a la aspiración de objetividad que planten a las demás ciencias" (citado por Lozano, 2006, p. 17). En consecuencia, la fenomenología busca "determinar el sentido dado a los fenómenos por la descripción e interpretación del discurso de quien los vivió" (Bautista, 2013, p. 55). Además, propone que "para el fenomenólogo, la conducta humana, lo que la gente dice y hace, es producto del modo en que define su mundo" (Taylor \& Bogdan, 1984, p. 23).

El Interaccionismo Simbólico: Es un paradigma que busca el estudio de la relación en los grupos de personas y su comportamiento, el cual según Blumer (1969) el interaccionismo simbólico está fundamentado en tres premisas principales, la primera en donde el ser humano orienta sus actos hacia las cosas en función de lo que estas significan para él. La segunda, es que el significado de estas cosas se deriva de, o surge como consecuencia de la interacción social que cada cual mantiene con el prójimo. Por último, tenemos que los significados se manipulan y modifican mediante un proceso interpretativo desarrollado por la persona al enfrentarse con las cosas que va hallando a su paso.

La Teoría Crítica: Es una forma de unión de diferentes corrientes del pensamiento, que tiene como objetivo reestructurar la teoría marxista, creando un modelo revolucionario y trasformador de observar la realidad. La teoría critica es concepto impulsado en el "rechazo a la justificación de la realidad sociohistórica presente por 
considerarla injusta y opresora (“irracional”), postulando en su lugar, la búsqueda de una nueva realidad más racional y humana" (Bautista, 2013, p. 55).

Posteriormente a la construcción del planteamiento del problema aparece los diseños investigativos más utilizados en la investigación cualitativa, ofreciendo una orientación a las personas sobre cómo pueden abordar la situación planteada y tomar la mejor decisión y el camino que más le convenga para llegar a la finalidad del proyecto investigativo, es así que para Hernández y Mendoza (2018) el "diseño en la ruta cualitativa es el abordaje general que se utilizará en el proceso de investigación”(p.524). Estos diseños se pueden clasificar en: 1. Teoría fundamentada, 2. diseños etnográficos, 2. diseños narrativos, 3. diseños fenomenológicos, 4. diseños de investigación-acción y 5 . estudios de caso.

1. Teoría fundamentada: Según Hernández y Mendoza (2018) "su propósito es inducir teoría basada en datos empíricos y se aplica a áreas específicas". Por lo tanto, el investigador elabora una respuesta o teoría en relación con un fenómeno, atribuidos a un ambiente en concreto y desde la posición de varios participantes. Además, este diseño investigativo es de gran utilidad cuando al consultar las teorías no explican de manera adecuada el fenómeno o planteamiento del problema, o cuando no explica todas sus partes. Este diseño fue formulado por a Glaser y Strauss (2006) el cual básicamente necesita que, para la construcción de una teoría en consecuencia de los datos, sus conceptos y las hipótesis estén sustentadas en un proceso sistemático articuladas con cada paso en la investigación. "Mientras que la verificación de las teorías ya existentes busca más bien su contrastación, la elaboración de una nueva teoría busca principalmente el hacerlo a partir de los datos" (Ruiz, 2012, p. 57). Este planteamiento teórico es un punto de inicio y una estructura en continuo desarrollo, por lo tanto, parte de un medio de teorizar sustantivo, "la característica definitoria de la teoría fundamentada es que las proposiciones teóricas no se postulan al inicio del estudio, sino que las generalizaciones emergen de los propios datos y no de forma previa a la recolección de los mismos" (Paz, 2003, p. 35).

2. Diseño etnográficos: La etnografía "es el arte y la ciencia de describir a un grupo humano, sus instituciones, comportamientos interpersonales, producciones materiales y creencias" (Angrosino, 2007, p. 35). De igual forma, es una práctica investigativa que se lleva a cabo en los entornos sociales, donde los etnógrafos recolectan información sobre la experiencia de las personas donde se analizan los patrones predecibles, para lo cual su método de campo se debe llevar en los entornos en donde habitan las personas, tomando en cuenta el contexto. Llevado a cabo mediante la interacción entre el investigador y las personas en estudio, siendo participantes y observadores del ambiente y sus integrantes. Al ser relacional requiere de un tiempo extenso determinado, es inductivo tomando detalles descriptivos para edificar patrones generales y es integral ya que se realiza un bosquejo completo del grupo en estudio, además "se procede a una búsqueda de patrones a partir de las cuidadosas observaciones del comportamiento vivido y de entrevistas detalladas con personas en la comunidad estudiada" (Angrosino, 2007, p. 34). Para Hernández y Mendoza (2018) la característica que debe tener el problema e investigación para ser tratado por el diseño es "cuando se pretende describir, entender y explicar un sistema social" (p.525). En conclusión, lo que busca la investigación etnográfica es entender la manera en que vive un grupo de personas partiendo desde la propia experiencia de los participantes. Por consiguiente "su meta es captar la visión de los nativos, su perspectiva del mundo que los rodea, el significado de sus acciones, de las situaciones que ellos viven y su relación con otras personas de la comunidad" (Bautista, 2013, p. 91). 
3. Diseño narrativos: Se enfoca en entender hechos, situaciones y eventos que vinculan sentimientos, emociones y pensamientos en relación con las experiencias relatadas por los participantes. Por lo cual son narraciones de sucesos y eventos que han sido registrados de diferentes maneras y contado por los intervinientes que vivieron el hecho. En los diseños narrativos la persona que investiga tiene presente el contexto y el sitio en que se presentan las experiencias, "reconstruye historias individuales, los hechos, la secuencia de eventos y los resultados e identifica categorías y temas en los datos narrativos para, finalmente, entretejerlos y armar una historia o narrativa general” (Hernández \& Mendoza, 2018, p. 548). Del mismo modo se entienden las historias de la vida como obras de origen social. "En su conformación concreta, se inspiran en narraciones culturales básicas y en historias de la vida ofrecidas por la cultura. La meta del análisis de los datos narrativos es más descubrir esos procesos constructivos y menos reconstruir procesos objetivos" (Flick, 2007, p. 220).

4. Diseño fenomenológicos: Tiene como padre al matemático Edmund Husserl (1859-1938), diseño que tiene como centro el comprender las experiencias ante un señalado fenómeno, que afecta a las personas en estudio y puede verse replicado en cada una. Debido a lo cual busca "determinar el sentido dado a los fenómenos por la descripción e interpretación del discurso de quien los vivenció, superando de esta forma la dicotomía cartesiana. Acceder a la esencia de los cuatro existenciales básicos: espacialidad, corporeidad, temporalidad y comunidad, y construir el mundo de la vida desde sus actores" (Bautista, 2013, p. 111). Por consiguiente, este método pretende focalizarse en el estudio de los fenómenos y vivencias en función de las opiniones y razones, entonces tiene un gran interés por hallar los significados que las personas de dan a los fenómenos desde una apostura cotidiana. De esta manera, "en la fenomenología los investigadores trabajan directamente las unidades o declaraciones de los participantes y sus vivencias, más que abstraerlas para crear un modelo basado en sus interpretaciones" (Hernández \& Mendoza, 2018, p. 548).

5. Diseños de investigación acción participativa: Este diseño fue aceptado como paradigma metodológico en 1983, la cual consiste en incluir a cada individuo de la comunidad destino. De tal forma que el diseño de investigación acción participativa vincula el proceso de intervención y la reforma social, por tal motivo es la misma población en estudio la que propone soluciones ante el problema que les atañe. Por lo tanto "es al mismo tiempo una metodología de investigación y un método de intervención social, por ser un movilizador social que genera tejido social" (Katayama, 2014, p. 61). Igualmente Sandín (2003, citado por Hernández \& Mendoza, 2018, p. 548) señala que "la investigación-acción pretende, esencialmente, propiciar el cambio social, transformar la realidad (social, educativa, económica, administrativa, etc.) y que las personas tomen conciencia de su papel en ese proceso de transformación" (p.552). Es por esta razón que se debe involucrar a las personas colaboradoras en el proceso de localización de las necesidades donde son estos los que conocen la realidad y el contexto.

6. Estudios de caso: Los estudios de caso son un diseño que busca la comprensión de un fenómeno en particular, para lo cual se efectúa un diagnóstico integral del objeto de estudio y en relación con este se establece el ambiente social que está presente. De tal manera que el estudio de caso se estudia cuando es evidente lo especial que tiene el hecho y lo relevante que puede llegar a ser. Para Stake (2007) "el estudio de caso es el estudio de la particularidad y de la complejidad de un caso singular, para llegar a comprender su actividad en circunstancias importantes" (p.57). Por lo tanto, su alcance es llegar a conocer a nivel profundo un fenómeno, donde se llega a la investigación a profundidad de un caso propuesto, es así que Hernández y Mendoza (2018) plantea que los "estudios que al utilizar los procesos de investigación 
cuantitativa, cualitativa o mixta analizan profundamente una unidad holística para responder al planteamiento del problema, probar hipótesis y apoyar el desarrollo de teoría" (p.185). De igual manera Ballestin y Fàbregues (2018) exponen que

"Un caso es un objeto de estudio con unas fronteras más o menos claras que se analiza en su contexto y que se considera relevante, ya sea para comprobar, ilustrar o construir una teoría o una parte de ella, o por su valor intrínseco"(p.83).

Figura 1.

Pilares de la Investigación cualitativa.
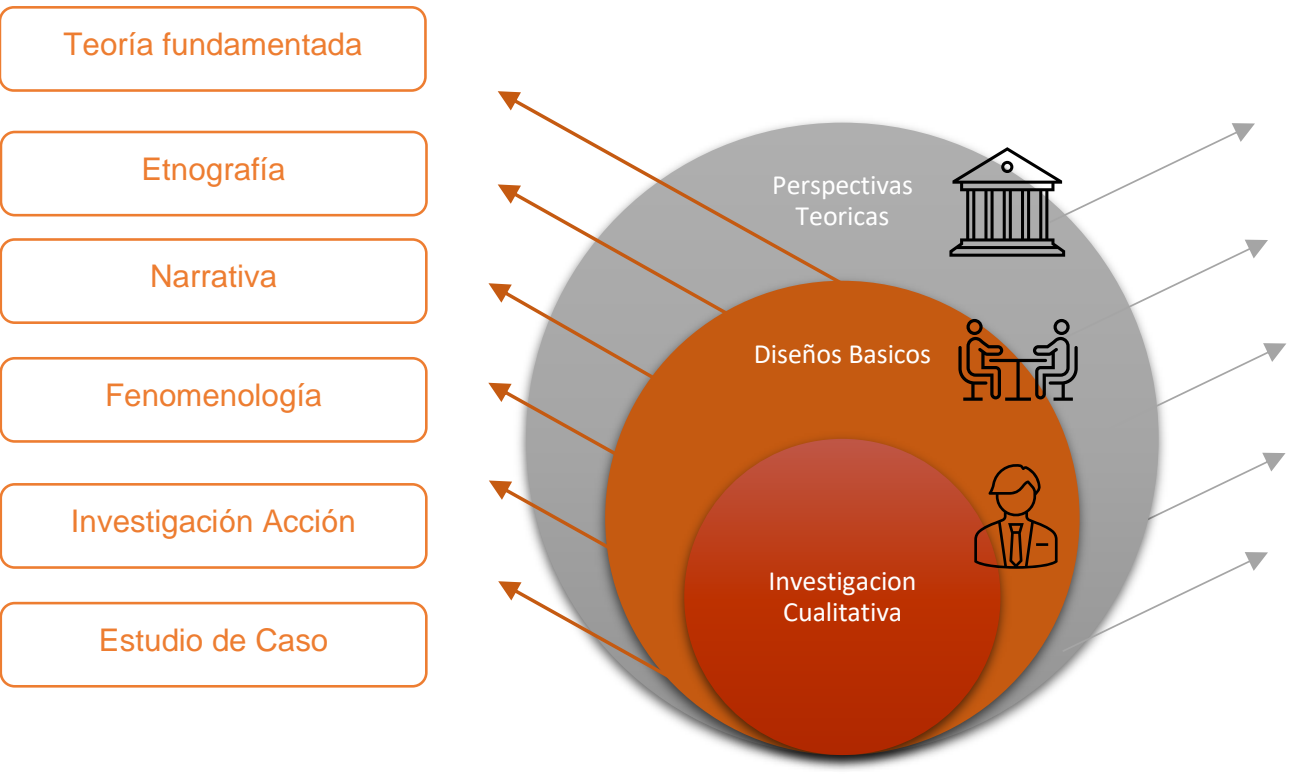

Interpretativismo

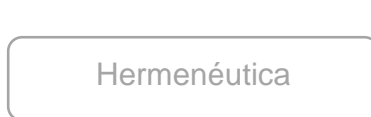

Fenomenología

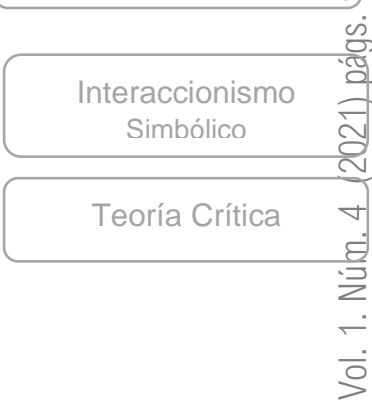

Nota: Enlace en la Investigación cualitativa de la Perspectiva teórica y los diseños básicos. Creación Propia.

Una de las dificultades que tiene la metodología cualitativa radica en su validez externa ya que su contenido no es generalizable a la población. De igual manera se propone que la corriente "no puede ser tan rígidamente planeada como para no replantearla durante el curso de esta. Una asesoría o dirección experta, y la crítica interna o externa, pueden convertirse en un termómetro para proceder" (Niño, 2011, p. 32). De la forma que, cuando queremos escoger la ruta de investigación adecuada para nuestro proyecto debemos según las recomendaciones de Hernández y Mendoza (2018) "de acuerdo con varios factores: el planteamiento del 
problema (lo que pretendamos indagar), los conocimientos que tengamos de cada método (ruta), las circunstancias particulares que rodean a la investigación (tiempo y recursos) y la propia experiencia" (p.16). En otro orden de ideas abrimos la puerta a una unión de metodologías de enfoque mixto donde la investigación cualitativa no llega a eliminar del todo los datos ya que estos "vienen a enriquecer y fundamentar los resultados del estudio. Estos dos enfoques, cuando están emparentados, permiten simplemente tener una visión más completa, más matizada y compleja de un fenómeno que se busca comprender" (Gómez,Deslauriers y Alzate, 2010,p.101). Por último, se diría que "la investigación cualitativa no es menos alérgica al número, así como la investigación cuantitativa no escapa al imponderable cualitativo" (Deslauriers, 2004, p. 2).

\section{CONCLUSIONES}

La investigación cualitativa es de gran manera inductiva, siendo la metodología utilizada en las ciencias sociales, al proveer de una estructura flexible al punto que el investigador se convierte en guía de la forma que realiza la conducción de sus estudios, del modo que cumple con los lineamientos orientadores, pero no está supeditado a reglas. Por ende, que el investigador observa el contexto y a los participantes desde una perspectiva holística, escudriñando en su ambiente, pasado y en las situaciones en que se encuentran. A su vez los observadores cualitativos llegan a identificarse con las personas que están estudiando con el propósito de comprender como viven las situaciones, por lo tanto, todas las miradas tienen y esconden una riqueza. Cuando estudiamos con este estilo podemos comprender sus vivencias a nivel personal y llegar a sentir de alguna forma las luchas cotidianas de las que hacen parte, de igual manera entendemos conceptos íntimos como la belleza y el dolor que no pueden ser notados con otros enfoques de investigación.

Entonces, la metodología cualitativa observa una parte de la realidad de manera subjetiva utilizando sus técnicas, estrategias e instrumentos concretos. Produciendo como resultado categorías conceptuales y una relación sistémica entre las partes y el todo de la realidad en estudio. En atención a lo cual la investigación con metodología cualitativa se mencione o no está provista de una estructura epistemológica y teórica que marca el camino especifico en la edificación del conocimiento y las posturas que se anclan en su construcción. Entonces al indagar sobre cada ruta esta nos señala los diseños, métodos, las técnicas e instrumentos de campo que debemos aplicar.

Por lo tanto, la congruencia que se encuentre entre las partes de la morfología de investigación será supeditada a la comprensión del investigador y de la finalidad del proceso investigativo, de esta manera se desprende la facilidad o dificultad a la hora de la toma de decisiones. En virtud de ello los diseños o métodos llamados por algunos autores son direcciones definidas los cuales forman marcos conceptuales que están unidos a diferentes teorías a partir de donde se establece la realidad en relación con determinados principios.

Esta investigación documental de carácter descriptivo sobre el método cualitativo abre la puerta para la comprensión de su estructura, tener clara la manera de proceder al escoger esta ruta, avanzar en la captación de seguidores, además tratar de concretar su estructura y aplicación en las ciencias sociales, siempre invitando a participar en su estudio y perfeccionamiento. Para concluir, es indispensable para realizar un trabajo investigativo con gran calidad que sean claras todas las piezas que están presentes en el tablero de la 
metodología cualitativa y que unidas son una herramienta magnifica para la comprensión de la complejidad humana.

\section{Conflicto de intereses / Competing interests:}

El autor declara que no incurre en conflictos de intereses.

Rol de los autores / Authors Roles:

No aplica.

\section{Fuentes de financiamiento / Funding:}

El autor declara que no recibió un fondo específico para esta investigación.

\section{Aspectos éticos / legales; Ethics / legals:}

El autor declara no haber incurrido en aspectos antiéticos, ni haber omitido aspectos legales en la realización de la investigación.

\section{REFERENCIAS}

Angrosino, M. (2007). Etnografía y observación participante en investigación cualitativa. Ediciones Morata (2. ed.). Morata.

Baena, G. (2017). Metodología de la investigación (3a. ed.). Grupo Editorial Patria.

Ballestin, B., \& Fàbregues, S. (2018). La práctica de la investigación cualitativa en ciencias sociales y de la educación. Editorial UOC.

Bautista, N. (2013). Proceso de la investigación cualitativa epistemología, metodología y aplicaciones. Resvista $\begin{array}{llll}\text { investigaciones en } & \text { educación, } & 13(2),\end{array}$ https://revistas.ufro.cl/ojs/index.php/educacion/article/view/1083

Bernal, C. (2010). Metodología de la investigación administración, economía, humanidades y ciencias sociales (Tercera ed). Prentice Hall.

Blumer, H. (1969). Symbolic interactionism: Perspective and methods. Englewood Cliffs, NJ: Prentice Hall.

Corbetta, P. (2007). Metodología y técnicas de investigación social. McGraw-Hill. https://diversidadlocal.files.wordpress.com/2012/09/metodologc3ada-y-tc3a9cnicas-deinvestigacic3b3n-social-piergiorgio-corbetta.pdf

Della, D., \& Keating, M. (2013). Enfoques y metodologías de las ciencias sociales una perspectiva pluralista.

Deslauriers, J. (2004). Investigación cualitativa: guía práctica. Papiro. http://repositorio.utp.edu.co/dspace/handle/11059/3365

Esterberg, K. (2002). Qualitative methods in social research. McGraw-Hill.

Flick, U. (2007). Introducción a investigación cualitativa. Ediciones Morata S. L.

Glaser, B., \& Strauss, A. (2006). The Discovery of Grounded Theory. Aldine'flr::.:msaction. http://www.sxf.uevora.pt/wp-content/uploads/2013/03/Glaser_1967.pdf 
Gómez, M., Deslauriers, J., \& Alzate, M. (2010). Cómo hacer tesis de maestría y doctorado. Investigación, escritura y publicación (1a. ed). Ecoe Ediciones.

González, M. (2015). Fuentes de Información. Universidad Autónoma de Hidalgo. https://repository.uaeh.edu.mx/bitstream/bitstream/handle/123456789/16700/LECT132.pdf

Hernández, R., Fernández, C., \& Baptista, M. (2014). Metodología de la investigación (Sexta edic). McGraw-Hill. http://observatorio.epacartagena.gov.co/wp-content/uploads/2017/08/metodologia-de-la-investigacionsexta-edicion.compressed.pdf

Hernández, R., \& Mendoza, C. (2018). Metodología de la investigación. Las rutas cuantitativa, cualitativa y mixta (Sexta edic).

McGraw-Hill. http://www.biblioteca.cij.gob.mx/Archivos/Materiales_de_consulta/Drogas_de_Abuso/Articulos/Sampieri LasRutas.pdf

Hurtado, J. (2000). Metodología de la investigación (Tercera ed). https://ayudacontextos.files.wordpress.com/2018/04/jacqueline-hurtado-de-barrera-metodologia-deinvestigacion-holistica.pdf

Katayama, R. (2014). Introducción a la investigación cualitativa: Fundamentos, métodos, estrategias y técnicas [Universidad Inca Garcilaso de la Vega]. http://hdl.handle.net/20.500.11818/559

Lozano, V. (2006). Hermenéutica y fenomenología. Husserl, Heidegger y Gadamer. EDICEP.

Niño, V. (2011). Metodología de la investigación. Diseño y ejecución (21 ed). Ediciones dela U.

Patton, M. (2011). Developmental evaluation: applying complexity concepts to enhance innovation and use. Guilford Press.

Paz, S. (2003). Investigación cualitativa en educación. fundamentos y tradiciones. Mc Graw Hill.

Pimienta, J., De la Orden, A., \& Estrada, R. (2012). Metodología de la investigación. Pearson.

Ruiz, J. (2012). Metodología de la investigación cualitativa (5ª). Universidad de Deusto Servicio de Publicaciones.

Sandoval, C. (2002). Investigación cualitativa. ARFO Editores e Impresores. https://panel.inkuba.com/sites/2/archivos/manual colombia cualitativo.pdf

Sherman, R., \& Webb, R. (2005). Qualitative research in education: Focus and methods. Taylor \& Francis eLibrary.

Stake, R. (2007). Investigación con estudio de casos. Ediciones Morata S. L.

Taylor, S., \& Bogdan, R. (1984). Introducción a los métodos cualitativos de investigación. Paidos. http://mastor.cl/blog/wp-content/uploads/2011/12/Introduccion-a-metodos-cualitativos-deinvestigación-Taylor-y-Bogdan.-344-pags-pdf.pdf 